\title{
Valoración de pacientes tratados con hemodiálisis según la Ley de Promoción de la Autonomía Personal y Atención a las personas en situación de dependencia
}

\author{
Lola Andreu Periz* - Carmen Moreno Arroyo* - Maricel Julve Ibañéz**
}

* Escuela de Enfermería. Universitat de Barcelona

** Servicio de Nefrología. Hospital Universitario de Bellvitge

\section{Resumen}

La valoración del grado de dependencia de los pacientes en diálisis ha sido objeto de interés como indicador clínico y organizativo. El objetivo del estudio es conocer el grado de dependencia que presentan las personas sometidas a tratamiento con diálisis en Catalunya, según los criterios de la Ley sobre Promoción de la Autonomía Personal y Atención a las personas en situación de dependencia.

Se ha realizado un estudio descriptivo transversal en 42 centros de Cataluña sobre el grado de dependencia de los pacientes en hemodiálisis. La recogida de datos se hizo mediante una encuesta basada en el baremo de la Ley más datos sociodemográfico y características del tratamiento.

De los pacientes que componían la población renal de Cataluña fueron considerados por los profesionales sanitarios con algún grado de dependencia 806, de ellos 425 eran hombres y 381 mujeres; un 61\% tienen edades superiores a 70 años. El 53\% viven en pareja y el $80,1 \%$ tenían uno o más hijos. El 77,4 $\%$ de los pacientes habían sido trabajadores no cualificados, y el $65,4 \%$ dijeron no tener estudios. Se consideraron no dependientes 137 pacientes, con dependencia moderada 350, con dependencia

Correspondencia:

Carmen Moreno Arroyo

Escuela de Enfermería. Universitat de Barcelona

C/ Feixa LLarga s/n

08907. L'Hospitalet de Llobregat. Barcelona importante 237 y con gran dependencia 82. Fue significativa $(p<0,005)$ la relación de la dependencia con la edad, la movilidad y en los grandes dependientes la incapacidad para tomar decisiones. No tuvo significación estadística el tiempo de permanencia en hemodiálisis.

Aunque el baremo de la Ley rebaja la valoración de dependencia realizada por los profesionales, los centros de hemodiálisis están atendiendo a un 19\% de pacientes dependientes

\section{PALABRAS CLAVE:}

- HEMODIÁLISIS

- CAPACIDAD FUNCIONAL

- DEPENDENCIA

- LEY SOBRE PROMOCIÓN DE LA AUTONOMÍA

PERSONAL Y ATENCIÓN A LAS PERSONAS

EN SITUACIÓN DE DEPENDENCIA

\section{Evaluation of patients treated with haemodialysis according to the Act for the Promotion of Personal Autonomy and Attention to Persons in a Situation of Dependency}

\section{Abstract}

The evaluation of the degree of dependency of patients on haemodialysis is a clinical and organizational indicator. The aim of the study is to determine the degree of dependency of people undergoing 
haemodialysis in Catalonia, according to the criteria established by the Act for the Promotion of Personal autonomy and Attention to Persons in a Situation of Dependency.

A transversal descriptive study was carried out in 42 centres in Catalonia on the degree of dependency of patients on haemodialysis. The data were collected through a survey based on the scale established by the Act plus sociodemographic data and treatment characteristics.

Of the patients who made up the renal population of Catalonia (3868), 806 were considered by the healthcare professionals to have a high degree of dependency, of whom 425 were men and 381 were women; $61 \%$ were older than $70.53 \%$ live with a partner and $80.1 \%$ had one or more children. $77.4 \%$ of the patients had been unskilled workers, and $65.4 \%$ stated that they had no educational qualifications. 137 patients were considered not dependent, 350 were considered to have moderate dependency, 237 with significant dependency and 82 with a high dependency. There was a significant $(p<0.005)$ relationship between dependency and age, mobility and, in those with high dependency, with the inability to make decisions. The time they had been on haemodialysis was not statistically significant.

Although the scale established by the Act reduces the evaluation of dependency carried out by the professionals, $19 \%$ of the patients being treated by the haemodialysis centres have a clear lack of personal autonomy.

\section{KEY WORDS:}

- HAEMODIALYSIS

- DEPENDENCY EVALUATION

- FUNCTIONAL AUTONOMY

- ACT FOR THE PROMOTION OF PERSONAL

AUTONOMY AND ATTENTION TO PERSONS

IN A SITUATION OF DEPENDENCY

\section{Introducción}

La enfermedad renal unida al proceso de envejecimiento esta asociada a cambios que disminuyen la capacidad funcional de las personas. Estas incapacidades también pueden percibirse en personas que aunque sean jóvenes, tengan además del deterioro del riñón, otras patologías, como la diabetes; si unimos todas estas circunstancias, edad, pluripatología y tratamiento con hemodiálisis el resultado puede ser devastador para la autonomía de la persona y le llevaran a una situación de gran dependencia que hará que la persona enferma necesite además de una atención estrictamente sanitaria de numerosos cuidados que satisfagan sus necesidades ${ }^{1-2}$.

Un aspecto común dentro del envejecimiento constituye el hecho de que, la edad no hace más que acentuar las carencias de cada persona, de modo que en muchos casos se necesite de la ayuda de terceras personas, tanto en el ámbito físico (sobre todo para las actividades básicas de la vida diaria), psicológico (especialmente emocional y afectivo), socioeconómico (entorno familiar, de la comunidad e instituciones), etc. Además de la edad, existen varios factores potencializadores de la dependencia: género, soledad, recursos sociales, nivel educativo, nivel de renta, hábitos de vida e índice subjetivo de los miedos y temores ${ }^{2}$.

La dependencia puede entenderse como el resultado de un proceso que se inicia con la aparición de un déficit en el funcionamiento corporal como consecuencia de una enfermedad o accidente. Este déficit comporta una limitación en la actividad. Cuando esta limitación no puede compensarse mediante la adaptación del entorno, provoca una restricción en la participación que se concreta en la dependencia de la ayuda de otras personas para realizar las actividades de la vida cotidiana. Las necesidades de estas personas abarcan muchos aspectos que implican a la familia y a los servicios sociales, supone unos costos personales y económicos importantes que siempre se han cubierto de forma insuficiente ${ }^{3,4}$.

Una de las mayores iniciativas para cubrir esta carencia, fue la promulgación de la Ley 39/2006, de 14 de diciembre, de Promoción de la Autonomía Personal y Atención a las personas en situación de dependencia que publicó el BOE del 5 de diciembre de 2006 y que 
fue respaldada por todas las fuerzas parlamentarias, aunque suscito un gran debate y en la actualidad continua siendo motivo de controversia por las dificultades que plantea su aplicación. A ésta Ley se la denomina más coloquialmente Ley de Dependencia (LD) y asume la definición de dependencia dada por el Consejo de Europa en su Recomendación N ${ }^{\circ}$ (98) 9 de 18 de septiembre de 1998 del Comité de Ministros como: la necesidad de ayuda o asistencia importante para las actividades de la vida cotidiana. Con ella se configura así un cuarto pilar- unido a la sanidad, la educación y las pensiones- de este Estado del Bienestar que aunque la coyuntura social y económica lo hace difícilmente viable, España pretende mantener y superar un sistema asistencial anticuado y colocarse entre los países más avanzados en asistencia social.

El gran esfuerzo social que en España implica el tratamiento de la insuficiencia renal seguirá aumentando, ya que se estima que la cifra de más de 40.000 personas que están en programas de diálisis o trasplante se ampliará en los próximos años debido al envejecimiento de la población y a la mayor prevalecía de procesos crónicos como la diabetes o la hipertensión. La atención sociosanitaria a estas personas necesita de una potente organización en cuanto a mecanismos de prevención, diagnóstico y tratamiento de la enfermedad $y$, aunque, numerosos indicadores avalan su calidad debemos estar preparados para asumir el reto que supone para las unidades de diálisis el hecho incontrovertible que cada vez son más las personas dependientes que deben tratarse en las unidades nefrológicas ${ }^{5-7}$.

La valoración del grado de dependencia de los pacientes en diálisis ha sido objeto de interés por parte de numerosos autores ya que el perfil actual de esta población, no solo tiene implicaciones directas sobre su morbimortalidad sino que también afecta de forma determinante a otros aspectos funcionales, como la cantidad y calidad de cuidados que precisan o de la propia estructura de las unidades para facilitarles la ayuda que necesitan ${ }^{8}$. La valoración del grado de autonomía y por tanto de su posible dependencia se ha hecho utilizando numerosos instrumentos como el Índice de Actividades de la vida diaria, Índice de Cartel, de Lawton o Test delta ${ }^{5}$; no obstante la Ley de Dependencia ha definido un baremo que aunque inspirado en los instrumentos citados es propio y al que deben ajustarse todos los Servicios Sociales para definir a las personas a valorar e incluirlas en el grado que les corresponde para poder hacerlas partícipes de los posibles beneficios que la Ley otorga. Por tanto, será necesario que también en la valoración clínica de nuestros pacientes utilicemos este baremo para definir la dependencia de nuestros pacientes.

\section{Objetivos}

- El objetivo de este estudio es conocer y analizar el grado de dependencia que presentan las personas sometidas a tratamiento con hemodiálisis en Catalunya, según los criterios establecidos por la Ley sobre Promoción de la Autonomía Personal y Atención a las personas en situación de dependencia.

- Así mismo, se propone definir la existencia de patrones comunes propios de esta situación y, eventualmente, definir cuales son las principales causas de dependencia sobre las que se puede actuar.

\section{Personas y Método}

Se ha realizado un estudio descriptivo transversal de la información sobre el grado de dependencia de los pacientes que realizan diálisis en su modalidad de hemodiálisis como tratamiento sustitutivo renal en Cataluña.

La recogida de datos se llevó a cabo mediante la cumplimentación de unas encuestas encaminadas a conocer datos sociodemográfico y características del tratamiento dialítico. Se realizaron entre octubre de 2008 y enero de 2009, en 42 de los 44 centros de diálisis que en Cataluña que atienden a la población de personas mayores de 18 años tratadas con hemodiálisis.

Se solicitó que respondieran a la encuesta todos los pacientes que, según los profesionales que los atendían, presentaban alguna limitación para llevar a cabo las actividades de la vida diaria, precisando por tanto de la ayuda de un cuidador. En el caso de que la persona tuviera dificultades cognitivas la encuesta se hizo con la ayuda de un familiar o de un profesional de enfermería y/o médico conocedor de la situación de la persona encuestada. De los pacientes que en el momento del estudio componían la población renal de Cataluña fueron considerados por los citados profesionales con algún grado de dependencia 810. 
En primer lugar se efectuó un análisis descriptivo para conocer el perfil del paciente. Se tuvo en cuenta para el paciente variables sociodemográficas como sexo, edad, estado civil y situación de convivencia. Se tuvo en cuenta el tiempo de tratamiento con hemodiálisis y características de dicho tratamiento. Para conocer el grado de dependencia se utilizó el baremo que establece la Ley sobre promoción de la Autonomía personal y Atención a las personas en situación de dependencia. Este baremo es un instrumento fundamental de la Ley, ya que determina qué personas son dependientes y en qué grado. Es un documento técnico para valorar las siguientes actividades y capacidades:

- Comer y beber

- Regulación de la micción/defecación

- Lavarse

- Otros cuidados corporales

- Vestirse

- Mantenimiento de la salud

- Transferencias corporales

- Desplazarse dentro y fuera del hogar

- Tomar decisiones

Cada una de las actividades para las que la persona necesita ayuda, recibe una puntuación que permite establecer una clasificación de niveles de autonomía personal y de necesidad de supervisión y/o apoyo para estas actividades lo que permite determinar el grado y nivel de dependencia de las personas. La persona recibe una evaluación de hasta 100 puntos que la definen como persona dependiente en los siguientes grados y niveles:

- Grado I: 25-49 puntos: Nivel 1: 25-39; Nivel 2: 4049

- Grado II: 50-74 puntos: Nivel 1: 50-64 puntos, Nivel 2: 65-74

- Grado III: 75-100 puntos: Nivel 1: 75-89, Nivel 2: 90-100

A todos los pacientes y para evitar sesgos de interpretación los entrevistó un único encuestador en el mismo centro donde recibían tratamiento, previamente se les se les explicó el motivo del estudio solicitando su participación y obteniendo su consentimiento. A continuación, se les asignó un código sólo conocido por el investigador principal y los valores obtenidos se introdujeron en el programa SPSS. 16 para su explotación. Las variables se expresan como media 0 porcentajes y para la comparación entre variables se utilizó la T de Studen ó el test no parámetrico de la U de Mann- White. Se estableció el nivel de confianza en un $p<0,05$.

\section{Resultados}

De los 810 pacientes 4 no pudieron ser entrevistados quedando la muestra definitiva de 806 pacientes, de ellos 425 eran hombres y 381 mujeres, destacando que un $61 \%$ tienen edades superiores a 70 años. En cuanto a su situación familiar el $53 \%$ viven en pareja, mientras que el $35,4 \%$ son viudos o separados; un $10,9 \%$ son separados. El 80,1\% tenían uno o más hijos. El $77,4 \%$ de los pacientes definieron haber tenido una actividad laboral como trabajador no cualificado o trabajador manual, así mismo el 65,4\% dijeron no tener estudios o tener estudios primarios. En cuanto al grado de dependencia los resultados pueden observarse en la tabla 1 . Fue significativa la relación de la dependencia con la edad $(p=0,037)$, la movilidad y en los grandes dependientes la incapacidad para tomar decisiones. No tuvo significación estadística el tiempo de permanencia en hemodiálisis $(p=0,77)$.

\begin{tabular}{|l|c|}
\hline No dependientes & 137 pacientes \\
\hline Dependientes grado 1 & 350 pacientes \\
\hline Dependientes grado 2 & 237 pacientes \\
\hline Dependientes grado 3 & 82 pacientes \\
\hline
\end{tabular}

Tabla 1: Grados de dependencia de los pacientes valorados

En la tabla 2 se observa la relación entre el grado de dependencia y diferentes variables relacionadas con el tratamiento dialítico.

Los aspectos evaluados por el baremo de la Ley mostraron que la mayor puntuación se relacionó con la movilidad y el $82 \%$ de los pacientes grado 2 y el $74,5 \%$ grado 1 necesitaban silla de ruedas o bastones para desplazarse. Así mismo todos los pacientes grado 2 necesitaban ayuda para las actividades de la vida diaria relacionadas con comer, vestirse, higiene personal, salir a la calle y realizar labores domésticas y el 67,8\% necesitaban ayuda para tomar decisiones. Los pacientes grado 3 se mostraron totalmente dependientes para cualquier actividad de la vida diarias y sólo un $16 \%$ eran capaces de tomar decisiones. 


\begin{tabular}{|c|c|c|c|c|c|c|c|c|}
\hline \multicolumn{7}{|c|}{ Grado de dependencia } \\
\hline No dependiente & \multicolumn{2}{|c|}{ Grado I } & \multicolumn{2}{|c|}{ Grado II } & \multicolumn{2}{c|}{ Grado III } & Total \\
\hline N & $\%$ & N & $\%$ & N & $\%$ & N & $\%$ & N \\
\hline
\end{tabular}

\begin{tabular}{|c|c|c|c|c|c|c|c|c|c|}
\hline \multicolumn{10}{|l|}{ Grupos de edad } \\
\hline 0-39 años & 5 & 27,8 & 7 & 38,9 & 4 & 22,2 & 2 & 11,1 & 18 \\
\hline 40-59 años & 20 & 29,4 & 22 & 32,4 & 17 & 25,0 & 9 & 13,2 & 68 \\
\hline 60-69 años & 15 & 14,6 & 45 & 43,7 & 34 & 33,0 & 9 & 8,7 & 103 \\
\hline 70-79 años & 57 & 19,6 & 123 & 42,3 & 81 & 27,8 & 30 & 10,3 & 291 \\
\hline >79 años & 40 & 12,8 & 136 & 43,5 & 105 & 33,5 & 32 & 10,2 & 313 \\
\hline \multicolumn{10}{|l|}{ Años en HD } \\
\hline$<1$ año & 40 & 25,5 & 60 & 38,2 & 43 & 27,4 & 14 & 8,9 & 157 \\
\hline 1-2 años & 27 & 19,9 & 53 & 39,0 & 48 & 35,3 & 8 & 5,9 & 136 \\
\hline 3-4 años & 37 & 16,9 & 93 & 42,5 & 65 & 29,7 & 24 & 11,0 & 219 \\
\hline$>4$ años & 31 & 11,7 & 119 & 44,9 & 81 & 30,6 & 34 & 12,8 & 265 \\
\hline \multicolumn{10}{|l|}{ Horas semanales } \\
\hline$<4$ horas & 36 & 13,0 & 122 & 44,0 & 83 & 30,0 & 36 & 13,0 & 277 \\
\hline 4 horas & 95 & 19,6 & 191 & 39,4 & 153 & 31,5 & 46 & 9,5 & 485 \\
\hline$>4$ horas & 6 & 14,3 & 21 & 50,0 & 10 & 23,8 & 5 & 11,9 & 42 \\
\hline \multicolumn{10}{|c|}{ Tipo de acceso vascular } \\
\hline Catéter percutáneo & 2 & 16,7 & 5 & 41,7 & 4 & 33,3 & 1 & 8,3 & 12 \\
\hline Catéter tunelizado & 30 & 10,9 & 107 & 39,1 & 99 & 36,1 & 38 & 13,9 & 274 \\
\hline Favi & 102 & 20,6 & 217 & 43,8 & 132 & 26,6 & 45 & 9,1 & 496 \\
\hline Injerto & 3 & 13,6 & 5 & 22,7 & 11 & 50,0 & 3 & 13,6 & 22 \\
\hline \multicolumn{10}{|l|}{ Hospitalizaciones } \\
\hline Ninguna vez & 63 & 23,0 & 124 & 45,3 & 75 & 27,4 & 12 & 4,4 & 274 \\
\hline $1 \mathrm{vez}$ & 54 & 18,9 & 127 & 44,4 & 85 & 29,7 & 20 & 7,0 & 286 \\
\hline 2 veces & 18 & 12,2 & 47 & 32,0 & 48 & 32,7 & 34 & 23,1 & 147 \\
\hline 3 o más veces & 2 & 2,5 & 33 & 41,8 & 25 & 31,6 & 19 & 24,1 & 79 \\
\hline
\end{tabular}

Tabla 2: Análisis bivariado del Grado de Dependencia según la edad y diferentes variables clínicas

\section{Discusión}

Ante todo cabe destacar que la percepción de la dependencia que tiene el personal sanitario no es acorde con lo que la Ley propone y más de un $15 \%$ de pacientes que ellos consideraban dependientes no lo eran según la Ley. Esto se debe probablemente a que el personal sanitario los valora en situaciones muy concretas (hospitalizaciones, finalizar el tratamiento) y que por otra parte los pacientes se sienten más vulnerables en la sala de hemodiálisis y magnifican sus déficit. Por otra parte, se ha de tener en cuenta la filosofía restrictiva de la Ley, ya que al comportar gasto social, pretende que sólo se beneficien de ella casos muy claros en cuanto a dependencia. Ambas circunstancias justificarían que estudios realizados con otros baremos muestran índices de dependencia mucho más altos 9 .
Pese a esto una elevada proporción de pacientes en hemodiálisis presenta algún tipo de dependencia, que básicamente es por una deficiencia física que les impide una movilización adecuada y requerir ayuda para actividades que se realizan en la sala de diálisis como comer vestirse o ponerse en la cama o sillón. Por otra parte, en nuestro estudio se ha detectado que, como otros autores han observado, muchos pacientes presentan deficiencia psíquica. Así, se ha descrito una elevada prevalencia de alteraciones cognitivas en pacientes ancianos en hemodiálisis, que llega a ser 7,4 veces mayor que en pacientes ancianos de la población general, lo cual parece estar estrechamente relacionado con la arteriosclerosis avanzada que presentan estos pacientes ${ }^{10}$. 
Como señalan otros autores tampoco en nuestro estudio ha habido significación estadística entre el tiempo de permanencia en hemodiálisis y el grado de dependencia y aunque se pudiera pensar que los pacientes que llevan más tiempo en hemodiálisis estuvieran en peores condiciones físicas, por el deterioro que la hemodiálisis conlleva, en realidad estos pacientes iniciaron tratamiento sustitutivo más jóvenes y en mejores condiciones, mientras que en la actualidad se han ampliado los criterios de inclusión y cada vez más se admiten en los programas de diálisis personas inicialmente más deterioradas ${ }^{10}$.

La utilización de accesos vasculares externos es mayor en personas con mayor dependencia, probablemente este hecho se deba al mal estado de su red vascular que impide la realización de una fístula convencional ${ }^{11}$. El deterioro cognitivo puede dificultar la supervivencia del catéter y obligar a los cuidadores a adoptar precauciones respecto a su mantenimiento.

Es necesario que en la planificación del trabajo en las unidades se tenga en cuenta que el perfil del paciente sometido a hemodiálisis es cada vez más complejo y que la dependencia ocasiona unas cargas de trabajo al persona médico y de enfermería que en general no se consideran y por tanto no se valoran ni se tiene en cuanta a la hora de dotar de recursos a esas unidades ${ }^{10,12}$.

La enfermera que debe trasladar a un paciente en silla de ruedas, ayudarse por otra o más personas para movilizarlo, darle de comer en la boca y vigilarlo constantemente para que no se autolesione en una sesión de diálisis que suelen ser tormentosa por las incidencias que se producen (hipotensiones, falta de flujo) probablemente no podrá reflejar en la documentación clínica ni una pequeña parte de sus cuidados. Se debería reflexionar sobre el desgaste físico y emocional que supone el cuidar a personas cada vez más deterioradas sin posibilidades de recuperación y con pocos recursos para hacerlo.

En conclusión, los centros de hemodiálisis están atendiendo a pacientes con un importante grado de dependencia, lo que supone una mayor carga de trabajo para el personal sanitario y muy especialmente el de enfermería por lo que se debe considerar esta circunstancia a la hora de planificar una adecuada atención a estos pacientes.

\section{Bibliografía}

1. Muñoz C, Martín A, Millán M. Tolerancia y calidad de vida en ancianos hemodiálisis. Rev Soc Esp Enferm Nefr. 2001; 14: 92-93.

2. Rebollo P, Álvarez-Ude F, Valdés C, Estébanez C, FAMIDIAL Study Group: Different evaluations of the health related quality of life in dialysis patients. J Nephrol. 2004; 17: 833-40.

3. Rubio Herrera. ¿Cuándo comienza la dependencia en el mayor? Geriátrika. 2003; 19(9-10):289-96.

4. Ministerio de Trabajo y Asuntos Sociales. Libro Blanco sobre la atención a las personas en situación de Dependencia en España. Capitulo 1, Bases demográficas, estimación, características y perfiles de las personas en situación de dependencia. 2005; 4-86.

5. Querejeta González, M. Discapacidad/ dependencia unificación de criterios de valoración y clasificación IMSERSO. Edita: Ministerio de Trabajo y Asuntos Sociales Secretaría de Estado de Servicios Sociales, Familias y Discapacidad IMSERSO. Madrid. 2004.

6. Asensio A, Ramos A, Núñez S. Factores pronósticos de mortalidad relacionados con el estado nutricional del anciano hospitalizado. Med Clin (Barc). 2004; 123: 370-3.

7. Palacios A. El modelo social de discapacidad: orígenes, caracterización y plasmación en la Convención Internacional sobre los Derechos de las Personas con Discapacidad CERMI Madrid. 2008.

8. Montorio I, Fernández de Trocóniz MI, Sánchez Colodrón M. Losada, M Dependencia y autonomía funcional en la vejez. La profecía que se autocumple. Rev Mult Gerontol. 2002; 12(2):61.

9. Gómez MA, Arqués A, Martos, J. Castellón A. La capacidad funcional y el apoyo social informal en un grupo de personas mayores institucionalizadas Rev Mult Gerontol. 2005; 15(2):85-91.

10. Alvarez Ude F. Factores asociados al estado de salud percibido de los pacientes en hemodiálisis crónica. Rev Soc Esp Enferm Nefr. 2001; 14: 64-68.

11. García Cortés MJ, Viedma G, Sánchez Perales MC et al. Acceso vascular permanente en pacientes de edad avanzada que inician hemodiálisis: ¿Fístula o catéter? Nefrología. 2005; 25 (3): 307-14.

12. Contreras Abad MD, Rivero Arellano MF, Jurado Torres MJ, Crespo Montero, R. Perfil actual del paciente en hemodialisis hospitalaria. Análisis de sus necesidades Rev Soc Esp Enferm Nefrol. 2004; 7 (1): 56-61. 


\section{belco}

\section{The rightot therrapy way}

$$
\therefore \therefore
$$

